\title{
CENSO COPROLÓGICO NO MUNICIPIO DE PERUIBE (LITORAL SUL DO ESTADO DE SÃO PAULO). REGISTRO DE CASOS AUTÓCTONES DE ESQUISTOSSOMOSE MANSONI (1)
}

\author{
Paulo de Toledo ARTIGAS (2) \\ Mário Demar PEREZ (3) \\ Domingos BAGGIO (2)
}

\begin{abstract}
Artigas, P. de T.; Perez, M. D. \& Baggio, D. - Censo coprológico no município de Peruibe (Litoral Sul do Estado de São Paulo). Registro de casos autóctones de esquistossomose mansoni. Rev. Saüde públ., S. Paulo, 3(2):141-147, dez. 1969.

Resumo - Foram apresentados os resultados dos exames coprológicos, para levantamento das parasitoses intestinais na população da zona urbana do município de Peruibe (litoral sul do Estado de São Paulo, Brasil). Foram constatados 29 casos de esquistossomose mansoni, sendo 7 considerados autóctones de Peruibe e 5 do litoral sul do Estado de São Paulo.
\end{abstract}

O município de Peruibe foi escolhido como local de interêsse para a realização, em 1966, da Primeira Jornada Científica do Centro Acadêmico da Faculdade de Farmácia e Bioquímica, pelo fato de ter sido constatado nessa cidade, poucos meses antes, um caso de infestação natural por Schistosoma mansoni, na pessoa de J.R.C.T., natural de São Paulo (SP) e com 12 anos de idade; caso diagnosticado na Cadeira de Parasitologia da referida Faculdade, com ovoscopia positiva.

A região em estudo foi, para fins de lenvantamento, dividida em zonas urbana e rural; para a constituição da zona rural foram escolhidas as quatro fazendas limítrofes com a cidade: São João, Santa Izabel, Icatum e Guarau.

Os dados obtidos na zona rural, devido a fortes chuvas que assolaram a região, tornando quase impraticável o acesso às duas últimas fazendas, foram insuficientes para fins estatísticos. Esta zona será objeto de nossa atenção em trabalhos posteriores, que deverão atingí-la no seu todo.

A zona urbana foi subdividida em quatro setores (I, II, III e IV); foram sor-

Recebido para publicação em 16-4-1969.

(1) Apresentado como nota prévía na Associação Paulista de Medicina, em sessão realizada em 4 de março de 1966, do Departamento de Higiene e Medicina Tropical.

(2) Da Cadeira de Parasitologia da Faculdade de Farmárcia e Bioquímica da USP — São Paulo, Brasil. .

(3) Da Cadeira de Parasitologia da Faculdade de Farmácia e Bioquímica da USP e do Instituto Butantan - São Paulo, Brasil. 
ARTIGAS, P. de T.; PEREZ, M. D. \& BAGGIO, D. - Censo coprológico no municiplo de Peruibe

(Litoral sul do Estado de São Paulo). Rev. Saúde públ., S. Paulo, 3(2):141-147, dez. 1969.

teadas $20 \%$ das casas da população fixa, o que corresponde a 550 indivíduos (em sua maioria operários não classificados e seus familiares) e dos quais por motivos vários, só 475 fizeram exames coprológicos.

\section{METODOS DE TRABALHO}

Como meios de diagnóstico de laboratório foram realizados exames de fezes e intradermorreação.

Cada amostra de fezes foi submetida a exame macroscópico e microscópico; êste constou de pesquisa direta e após enriquecimento. Como técnica de enriquecimento foram utilizadas as de Willis (flutuação), de Faust (centrifugação e flu. tuação) e de Hoffman, Pons e Janner (sedimentação).

Considerando-se que, para cada amos. tra, foram preparadas sete lâminas (sendo três exames de sedimento) examinaram-se cêrca de 3.500 preparações, levando-se em conta as provas repetidas para confirmação.

A intradermorreação foi praticada com antígeno preparado por Perez, no setor de "Esquistossomose Experimental" do Instituto Butantan, segundo técnica modificada por RuIz ${ }^{2}$ (1953) — partida $02 / 62$.

\section{RESULTA DOS}

1 - A taxa da positividade de indivíduos portadores de parasitoses intestinais foi de $95,3 \%$, sendo as seguintes as taxas percentuais específicas:

$$
\begin{aligned}
& \text { A - Por Protozoários } \\
& 1 \text { - Entamoeba histolytica } \\
& 2 \text { - Giardia lamblia } \\
& 3 \text { - Entamoeba coli ... } \\
& 4 \text { - Iodamoeba butschilii } \\
& 5 \text { - Endolimax nana ... } \\
& 6 \text { - Chilomastix mesnili }
\end{aligned}
$$

$0,4 \%$

$3,3 \%$

$12,2 \%$

$1,6 \%$

$0,2 \%$

$0,2 \%$
B - Por Helmintos

1 - Schistosoma mansoni . $\quad 5,8 \%$

2 - Strongyloides stercoralis $\quad 12,0 \%$

3 - Ancylostomidae ... $\quad 73,4 \%$

$4-$ Ascaris lumbricoides $\quad 74,1 \%$

5 - Trichuris trichiura . $\quad 84,8 \%$

Nas Tabelas 1 e 2 figura a distribuição de protozoários e de helmintos, respectivamente, segundo grupos etárins e sexo dos indivíduos parasitados.

A ausência de ovos de Enterobius vermicularis nos resultados, deve decorrer do fato de não terem sido utilizadas técnicas eletivas, enquanto que a de Taenia talvez tenha como causa o baixo padrão de vida da população, que só excepcionalmente alimenta-se de carne bovina ou ovina, conforme dados obtidos no inquérito familiar realizado durante a Jornada; são essencialmente consumidores de carne de peixe.

2 - Em 119 indivíduos, moradores nos locais em que foram constatados casos positivos para Schistosoma mansoni, foi feita a intradermorreação para o diagnóstico de esquistossomose mansônica.

Baseados em dados anamenésticos, relativamente aos casos positivos para $S$. mansoni, sete foram considerados autóctones de Peruibe; cinco outros casos positivos tanto podem ser considerados autóctones de Peruibe, como de Itanhaem uns, ou de Peruibe, Registro, Itariri ou São Vicente outros. Um menor de 16 anos, natural e procedente de Volta Redonda, Estado do Rio de Janeiro, teve os exames de fezes negativos e a intradermorreação positiva; rotulamos provisòriamente como caso suspeito.

Os casos restantes, em número de 16 , são de indivíduos procedentes do Nordeste (Estado de Pernambuco, Alagoas, Sergipe e Ceará) e da região Leste do país (Estado de Minas Gerais e Bahia). Ver Tabelas 3,4 e 5 . 
ARTIGAS, P. de T.; PEREZ, M. D. \& BAGGIO, D. - Censo coprológico no municipio de Perulbe (Litoral sul do Estado de São Paulo). Rev. Saúde puibl., S. Paulo, 3(2):141-147, dez. 1969.

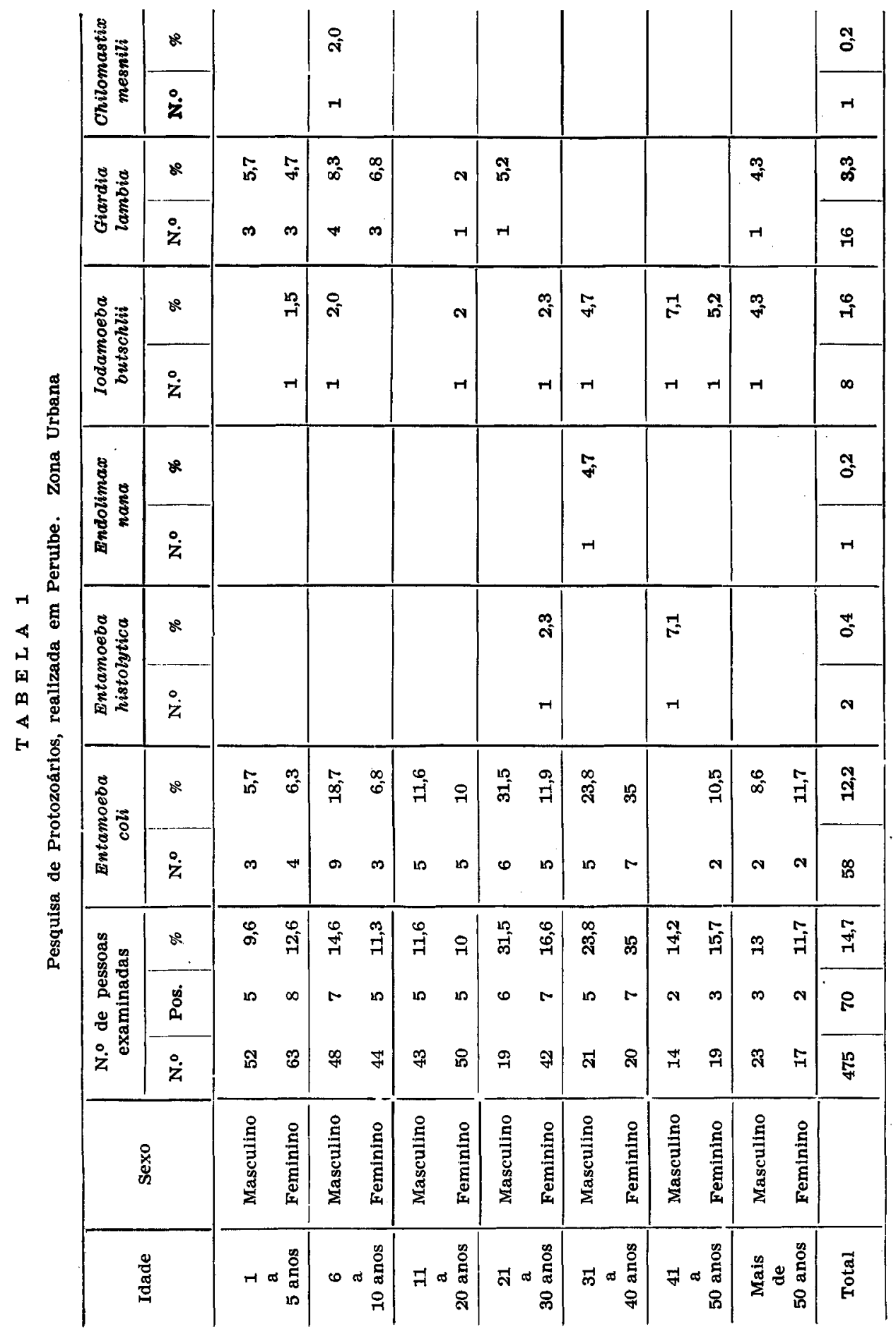




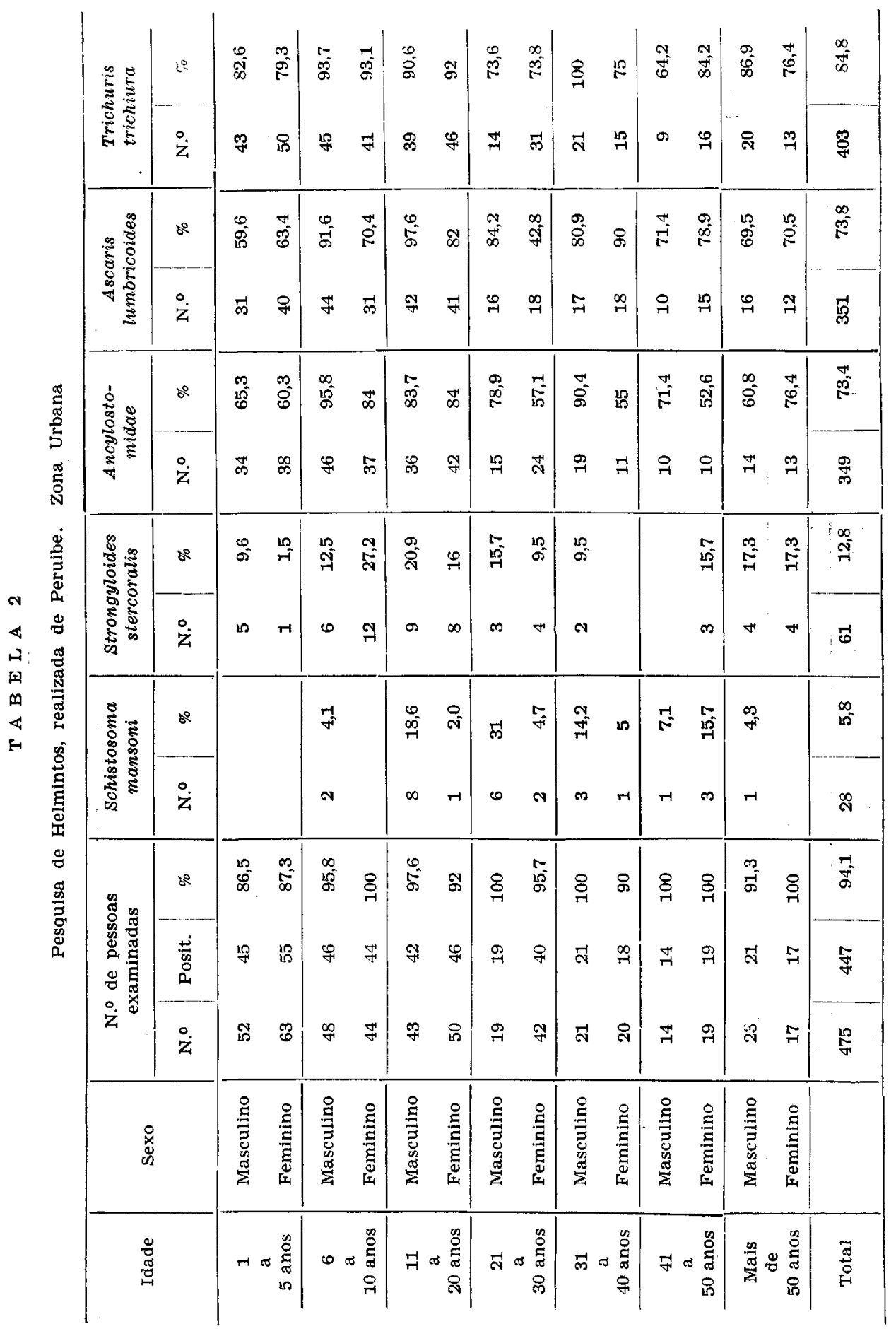


ARTIGAS, P. de T.; PEREZ, M. D. BAGGIO, D. - Censo coprológico no município de Perúbe (Litoral sul do Estado de São Paulo). Rev. Saúde públ., S. Paulo, 3(2):141-147, dez. 1969.

T A B E L A 3

Casos Autóctones de Peruibe

\begin{tabular}{l|c|c|c|c|c|c|c}
\hline Nome & $\begin{array}{c}\text { Natural } \\
\text { de }\end{array}$ & Sexo & Côr & Idade & $\begin{array}{c}\text { Tempo } \\
\text { de } \\
\text { Residencia }\end{array}$ & Fezes & I. R. \\
P.R.C. & Peruibe & Masculino & Parda & 13 anos & 13 anos & + & - \\
J.R.C. & Peruibe & Masculino & Parda & 12 anos & 12 anos & + & - \\
L.R.C. & Peruibe & Masculino & Parda & 10 anos & 10 anos & + & - \\
A.G.S.F. & Peruibe & Masculino & Parda & 17 anos & 17 anos & + \\
T.A.A. & Peruibe & Masculino & Parda & 30 anos & 30 anos & + \\
A.A.A. & Peruibe & Masculino & Parda & 12 anos & 12 anos & + \\
R.A.A. & Peruibe & Masculino & Parda & 11 anos & 11 anos & + \\
\hline
\end{tabular}

(*) Năo foi feita.

T A B E L A 4

Outros Casos Autóctones do Litoral Sul do Estado de São Paulo

\begin{tabular}{|c|c|c|c|c|c|c|c|}
\hline Nome & Natural & Sexo & Côr & Idade & Procedência & Fezes & I. $\mathbf{R}$. \\
\hline J.D.R. & Itanhaem & Masculino & Branca & 13 anos & Itanhaem & + & $(*)$ \\
\hline J.R.S.P. & S. Vicente & + Masculino & Parda & 12 anos & S. Vicente & + & - \\
\hline J.C. & Registro & Masculino & Branca & 35 anos & Itariri & + & + \\
\hline M.H.S. & Itariri & Feminino & Branca & 11 anos & Itariri & + & - \\
\hline I.D.A. & S. Vicente & Masculino & Branca & 13 anos & S. Vicente & + & - \\
\hline
\end{tabular}

(*) Não foi feita, 
ARTigas, P. de T.; PEREZ, M. D. \& BAGgio, D. - Censo coprológico no municipio de Peruibe (Litoral sul do Estado de São Paulo). Rev. Saúde públ., S. Paulo, 3(2):141-147, dez. 1969.

T A B E L A 5

Casos positivos para Schistosoma mansoni. Resultados dos exames de fezes confrontados com os da I.R. em individuos, submetidos a essas duas provas, procedentes das regloes Leste e Nordeste do pais

\begin{tabular}{|c|c|c|c|c|c|c|}
\hline Nome & $\begin{array}{c}\text { Natural } \\
\text { de }\end{array}$ & Sexo & Cór & Idade & Fezes & $\begin{array}{l}\text { Intrader- } \\
\text { morreaçăo }\end{array}$ \\
\hline M.F.A. & Ceará & Feminino & Branca & 42 anos & + & + \\
\hline L.E.S. & Minas Gerais & Feminino & Preta & 45 anos & + & + \\
\hline E.F.S. & Minas Gerais & Masculino & Preta & 21 anos & $t$ & + \\
\hline A.G. & Bahia & Feminino & Parda & 46 anos & + & $(*)$ \\
\hline A.F.M. & Bahia & Masculino & Branca & 38 anos & + & + \\
\hline D.V.S. & Minas Gerais & Feminino & Parda & 25 anos & $t$ & + \\
\hline N.M. & Minas Gerais & Masculino & Branca & 9 anos & + & suspeita \\
\hline J.S.S. & Pernambuco & Masculino & Branca & 30 anos & + & $(*)$ \\
\hline A.L.S. & Alagoas & Feminino & Branca & 22 anos & + & + \\
\hline E.D.S. & Sergipe & Masculino & Parda & 58 anos & + & $(*)$ \\
\hline E.C. & Sergipe & Feminino & Parda & 38 anos & + & + \\
\hline J.R.S. & Sergipe & Masculino & Branca & 41 anos & + & $(*)$ \\
\hline A.P.S. & Bahia & Masculino & Parda & 25 anos & + & (*) \\
\hline M.S.S. & Bahia & Feminino & Parda & 23 anos & + & + \\
\hline G.A.S. & Bahia & Masculino & Parda & 34 anos & + & $(*)$ \\
\hline H.A.S. & Bahia & Feminino & Parda & 14 anos & - & + \\
\hline J.J.A. & Bahia & Masculino & Parda & 25 anos & + & + \\
\hline
\end{tabular}

(*) Năo fol feita.

Paralelamente a êste Censo, foram realizadas coletas de planorbídeos para identificação e verificação de focos com moluscos portadores de forma evolutiva do Schistosoma mansoni; os resultados de tais pesquisas foram apresentados por $\mathrm{PE}$ -
REz \& ArTigas ${ }^{1}$ (1966), como nota prévia, em sessão do Departamento de $\mathrm{Hi}$ giene e Medicina Tropical, da Associação Paulista de Medicina e estão sendo publicados em trabalho à parte. 
ARTIGAS, P. de T.; PEREZ, M. D. \& BAGGIO, D. - Censo coprológico no municiplo de Peruibe (Litoral sul do Estado de São Paulo). Rev. Saúde públ., S. Paulo, 3(2):141-147, dez. 1969.

\section{DIS C U S A O}

Os resultados acima referidos confirmam as previsões sôbre a existência de focos de esquistossomose em Peruibe, evento previsto desde a verificação do caso de J.R.C.T.

Assinale-se que, nas vésperas do início da Jornada, os autores tiveram conhecimento de que o Departamento do Serviço do Interior da Secretaria da Saúde Pública da Assistência Social, já tinha registrado casos de esquistossomose man. soni autóctones de Peruibe, não entregues a publicidade.

\section{A G R A D E C I M E N T O}

Ao Centro Acadêmico da Faculdade de Farmácia e Bioquímica que patrocinou e participou dessa Jornada, numa destacada demonstração de interêsse social e de aplicação de conhecimentos auferidos no curso escolar.

Ao Sr. Santo Limberto, técnico da Cadeira de Parasitologia que, atendendo nosso convite, colaborou na realização dos exames parasitológicos.

Artigas, P. de T.; Perez, M. D. \& Baggro, D. - Coprologic census of Peruibe (Southern Town in the coast of the State of São Paulo). Record of autochthonous cases of manson's schistosomiasis. Rev. Saúde pribl., S. Paulo, 3(2) :141-147, dez. 1969.

Summary - The results of feces examinations, in order to know the degree and variation of intestinal parasitoses among the inhabitants of Peruibe, a littorean city in the south of the State of Săo Paulo, Brazil, are presented. Among 475 persons, children and adults, living in $20 \%$ of the houses of the town, $95.3 \%$ proved to be parasited. The percentual results of helminths and protozoa infestations are related in tables. Seven autochthonous cases of manson's schistomiasis have been discovered, i.e., a percentage of $5.89 \%$.

\section{REFERENCIAS BIBLIOGRAFICAS}

1. PEREZ, M. D. \& ARTIGAS, P. T. Contribuicão ao levantamento da Carta Planorbidica do Estado de Săo Paulo. Pesquisa de focos com formas evolutivas do Schistosoma mansoni. II. Municipio de Perulbe (litoral sul do Estado de Săo Paulo). Nota prévia. Rev. paul. Med., 68:56, Jul. 1966 .

2. RUIZ, J. M. - Preparo do antigeno para intradermorreacão na esquistossomose. Mem. Inst. Butantan, 25:5-14, 1953. 